\title{
ETNOGRAFIA COMO ESTRATÉGIA INVESTIGATIVA DA CULTURA ORGANIZACIONAL PARA A SUSTENTABILIDADE
}

\author{
Gabriela Almeida Marcon \\ Mestranda em Administração \\ Universidade Federal de Santa Catarina - UFSC \\ gabriela@almeidamarcon.com
}

Eduardo Juan Soriano-Sierra

Doutor em Oceanografia Biológica

Universidade Federal de Santa Catarina - UFSC

eduardo.soriano.sierra@gmail.com

\section{RESUMO}

O conhecimento de capacidades gerenciais atreladas ao desenvolvimento sustentável adquiriu importância nos últimos tempos para as mais diversas áreas. O conceito de cultura organizacional tornou-se relevante no bojo da literatura acerca da sustentabilidade. Em diversos relatórios políticos, a educação é descrita como uma ferramenta para a promoção do desenvolvimento sustentável. Uma nova cultura organizacional depende da aquisição e do compartilhamento de novos conhecimentos. Há grande oportunidade para pesquisa sobre cultura organizacional voltada à sustentabilidade, uma vez que os estudos existentes ainda são escassos. A pesquisa qualitativa apresenta-se como abordagem mais adequada ao levantamento de aspectos culturais. Este artigo buscou examinar as características da observação etnográfica a fim de verificar sua adequação para investigar e caracterizar uma cultura organizacional orientada para a sustentabilidade. Para fins de aplicação da etnografia para investigação da cultura organizacional, recomenda-se o estabelecimento de duas premissas epistemológicas. Limitou-se a analisar a adequação da etnográfica como estratégia investigativa da cultura organizacional voltada à sustentabilidade. Esse artigo não buscou mapear uma cultura de sustentabilidade, suas características e as prescrições para uma transformação cultural. Conclui-se que a etnografia é adequada à proposta por sua própria natureza, uma vez que dela resulta uma descrição densa do fenômeno.

Palavras-chave: Cultura organizacional; Etnografia, Gestão do conhecimento; Sustentabilidade.

\section{ETHNOGRAPHY AS INVESTIGATIVE STRATEGY OF ORGANIZATIONAL CULTURE TOWARDS SUSTAINABILITY}

\section{ABSTRACT}

The knowledge of management skills regarding sustainable development gained importance in recent times in several areas. The concept of organizational culture has acquired importance on the academic literature. In many political reports, education is described as a tool for promoting sustainable development. A new organizational culture depends on the knowledge acquisition and sharing. There is great opportunity for research on organizational culture towards sustainability, since existing studies are still scarce. Qualitative research is presented as the most appropriate approach to raise cultural aspects. This paper aims to examine the characteristics of ethnographic observation to verify whether it is the appropriate approach to investigate and characterize organizational culture for sustainability. To apply ethnography as research method for the organizational culture, it is recommended to establish two epistemological premises. It was limited to analyzing ethnographic research as a proper method to investigate sustainability. The paper did not aim to map a culture of sustainability, its characteristics and the requirements for a cultural transformation. It concludes that ethnography is suitable to the proposal by its very nature, since it results in a dense description of the phenomenon.

Key words: Ethnography; Knowledge management; Organizational culture; Sustainability. 
Etnografia como estratégia investigativa da cultura organizacional para a sustentabilidade.

\section{INTRODUÇÃO}

Sustentabilidade é um dos temas mais debatidos dos últimos tempos (Murphy \& Poist, 1995; Erturgut \& Soysekerci, 2009). Recentemente, o conhecimento e as capacidades gerenciais atreladas à sustentabilidade corporativa tornaram-se elementos cada vez mais significativos para as mais diversas carreiras (Hesselbarth \&Schaltegger, 2014). Em vários relatórios políticos, a educação é descrita como uma das ferramentas mais poderosas para formar indivíduos com as habilidades, competências e atitudes de consumidores sustentáveis (Oecd, 2008). O conceito de cultura organizacional tornou-se popular dentro da literatura de sustentabilidade (Linnenluecke \& Griffiths, 2010). O último relatório do Fórum Econômico Mundial demonstra que é crescente a preocupação com riscos globais decorrentes de questões socioambientais (WEF, 2016).

Uma nova cultura depende da aquisição e do compartilhamento de novos conhecimentos e experiências, do estabelecimento de novos fluxos - compreendidos como produção e propagação do conhecimento por toda a organização (Zhuge, 2006) - e, sobretudo, perpassa a sensibilização e internalização destes saberes pelos membros do sistema.

$\mathrm{Na}$ era do conhecimento, é necessário que as instituições desenvolvam uma cultura de sustentabilidade para que, conscientes da importância das boas práticas ambientais, adotem novas posturas. O paradigma racionalista, que marcou a industrialização em todo o mundo, negligenciando a subjetividade humana e a dependência da qualidade do meio ambiente para a manutenção da dignidade dos indivíduos, culminou na crise de sustentabilidade hodiernamente enfrentada (Lozano, 2011). Apesar disto, há pouca base teórica qualificada sobre o que realmente constitui uma cultura organizacional orientada para a sustentabilidade, uma vez que as prescrições para uma transformação cultural existentes são genéricas e reducionistas (Linnenluecke \& Griffiths, 2010; Harris \& Crane, 2002). Isto se afigura como oportunidade de pesquisa.

É por meio da pesquisa qualitativa que se pode examinar separadamente as partes para compreender o todo sistêmico, isto porque o sentido do todo está congregado na experiência vivida dos sujeitos objeto do estudo, vistos da perspectiva do pesquisador (Merriam, 1998). De acordo com as abordagens qualitativas, a etnografia parece a mais adequada para desbravar a experiência disseminada na cultura organizacional, as práticas sociais e os sentimentos humanos relativos a elas. Ao dividir algum tempo com certo grupo social, o pesquisador tem a oportunidade de observar comportamentos que dificilmente seriam mencionados abertamente pela comunidade sob estudo (Malinowski, 1984).

A etnografia aparenta ser o método ideal para compreensão da cultura da sustentabilidade. É necessário que as organizações desenvolvam uma cultura de sustentabilidade para que se adequem às novas necessidades da sociedade, na qual as ameaças socioambientais são preocupantes, o conhecimento é rapidamente construído e difundido, bem como os riscos sociais e ecológicos. A ênfase na estratégia ambiental implica a adoção de medidas aptas a evitar ou reduzir danos ecológicos ocasionados pelas atividades empresariais (Dicle \& Kose, 2014). No setor público, a sustentabilidade ambiental e a responsabilidade social são demandas fundamentais que devem prevalecer até mesmo sobre o desempenho econômico (Merad et al., 2014). Neste contexto, busca-se responder à seguinte questão: Como investigar e caracterizar uma cultura organizacional orientada para a sustentabilidade por meio da abordagem etnográfica?

Esta pesquisa busca, como objetivo geral, examinar as características da observação etnográfica a fim de verificar sua adequação para investigar e caracterizar uma cultura organizacional orientada para a sustentabilidade. Para tanto, o artigo contempla os seguintes objetivos específicos: a) elaborar uma revisão sistemática da literatura a fim de distinguir a pesquisa qualitativa da pesquisa quantitativa e identificar o conceito de etnografia; b) enquadrar a pesquisa etnográfica, dentre as abordagens qualitativas, particularizando-a; c) examinar a etnografia como estratégia para observar aspectos culturais. O propósito limita-se a analisar a pesquisa etnográfica como forma adequada a investigar uma cultura. O artigo não busca mapear uma cultura de sustentabilidade, suas características e as prescrições para uma transformação cultural. Neste 
momento se quer saber se a etnografia pode satisfatoriamente servir ao propósito de, em pesquisas futuras, desbravar uma cultura orientada para a sustentabilidade.

Esse artigo está estruturado da seguinte forma: além desta introdução, as seções seguintes apresentam o referencial teórico, método, discussão dos resultados e conclusões do estudo. Esta pesquisa justifica-se pela relevância, originalidade e viabilidade (Castro, 1977), além da contribuição à academia em futuros estudos acerca das capacidades dinâmicas atreladas à promoção da sustentabilidade nas organizações.

\section{REFERENCIAL TEÓRICO}

\subsection{A cultura da sustentabilidade nas organizações}

A sustentabilidade pode ser conceituada como estado das coisas que resulta do processo de desenvolvimento sustentável, que consiste em usufruir no presente sem deixar de assegurar às gerações futuras recursos suficientes à sadia qualidade de vida (Wced, 1987; Giddings et al., 2002; Sneddon et al., 2006; Benn \& Kearins, 2012). A sustentabilidade de uma organização depende, sobretudo, das características essenciais internas e externas desta organização que merecem ou não serem preservadas ao longo do tempo e, ainda, da definição do que é considerado um estado adequado em médio e longo prazo (Merad et al., 2014).

Ao longo do tempo, os países firmaram compromissos visando à inserção do meio ambiente e do desenvolvimento socioeconômico no centro da tomada de decisões políticas e econômicas (Sitarz, 1993; Morel et al, 2004; Vieira, 2012; WEF, 2016). O processo de sensibilização das lideranças mundiais, porém, é lento. A sociedade está cada vez mais atenta às questões ambientais. A preocupação com responsabilidade social deve começar no ambiente interno, podendo trazer ganhos estratégicos aos gestores (Burke \& Longsdon, 1996; Banerjee, 2002; Gomes, 2010; Porter \& Kramer, 2011; Dicle \& Kose, 2014; Vidal-Salazar et al., 2012). Grande parte do trabalho relativo à transição para a efetiva sustentabilidade ambiental demandará investimentos em infraestrutura e novos projetos para saúde e educação (Pnud, 2015).

Reivindicações mais intransigentes dos consumidores, legislações mais rigorosas e restrições de recursos também podem ajudar a acelerar a adaptação da indústria às demandas por uma sociedade mais sustentável, fomentando uma cultura da sustentabilidade (Ammenberg \& Sundin, 2005; Spangenberg et al, 2010; Testa \& Iraldo, 2010; Hallstedt et al, 2013).

Pensar e agir de forma sustentável não é sempre algo natural. Na tentativa de lidar com a complexidade do ambiente, as organizações têm buscado fazer das ações de sustentabilidade uma capacidade dinâmica, integrada com as estratégias e modelos de negócios (Leonidou et al, 2015; Schrettle et al, 2014; Beske, 2012; Peters et al., 2011; Barba-Sanchez e Atienza-Sahuquillo, 2010; Reuter et al, 2010; Russo, 2003; Liboni et al., 2016). Em muitas instituições, há a preocupação em ecologizar e alterar processos internos de parte dos próprios colaboradores e usuários, não da alta gestão (v. g. Wals, 2014). A introdução de novas políticas e práticas sustentáveis em uma organização pode ser vista como um conjunto de mudanças organizacionais, demandando um processo de aprendizagem organizacional no curso do tempo, a fim de viabilizar a internalização de novos conceitos e o compartilhamento de novos conhecimentos (Maon et al., 2009; Hotternrott et al., 2016).

Em muitos casos, a adoção de práticas sustentáveis demanda uma mudança de cultura, desde que se considere cultura como complexo que abarca o conhecimento, as crenças, a arte, a compreensão da ética, a moral, as leis, os costumes, hábitos e as habilidades desenvolvidas pelos membros de uma comunidade (Tylor, 1871).

Para Gertz (1973), cultura denota um padrão historicamente transmitido de significados incorporados em símbolos. A cultura seria um sistema de concepções herdadas, expressas em formas simbólicas por meio das quais os seres humanos se comunicam, perpetuam e desenvolvem seus conhecimentos e atitudes em relação à própria vida. Ainda, é possível compreender cultura 
como aquilo que é necessário saber ou crer para conviver em determinada sociedade (Kahn, 1975). A cultura organizacional, por conseguinte, é o conjunto de valores compartilhados pelos membros da organização (Çakar \& Ertürk, 2010). No mesmo norte, James et al. (2008) definem a cultura organizacional como sistema de crenças, normativos, expectativas e comportamentos compartilhados, isto é, sistema de valores e normas de uma organização. Este conjunto, invariavelmente, sofrerá influência dos valores comungados por cada membro da organização em seu contexto sociocultural específico.

O interesse pela cultura das organizações cresce a partir do momento em que se verifica que esta está atrelada ao desempenho organizacional (Aidar et al., 2002). Uma transformação cultural passa também pela formação profissional, razão pela qual é fundamental ressaltar o papel das instituições de ensino superior no tocante à educação para o desenvolvimento sustentável (Shriberg, 2002; Moore, 2005; Nicolaides, 2006; Benn et al., 2013; Adomßent et al. 2014; Godemann et al., 2014; Hussein et al., 2014). Numa perspectiva global, inovações industriais atreladas a incentivos por parte da sociedade são fatores críticos na transformação social rumo a melhores práticas de sustentabilidade (Tukker \& Jansen, 2006; Petala et. al., 2010; Hallstedt et al, 2013).

Para que a organização desenvolva estratégias sustentáveis, é necessário que tenha abertura à inovação (Vidal-Salazar et al., 2012). A Gestão do Conhecimento, como processo de aquisição, recuperação e proteção dos saberes organizacionais (Benjamins, 2013; Gaines, 2013; AussenacGilles \& Gandon, 2013), auxilia a difusão da cultura da sustentabilidade, uma vez que se trata de uma abordagem resoluta e sistemática a fim de assegurar a plena utilização, não somente da base de conhecimento da organização, mas também das competências e habilidades das pessoas, dos pensamentos, das inovações e ideias, com o propósito de aumentar os níveis de eficiência e eficácia organizacionais (Dalkir, 2005).

\subsection{Métodos qualitativos, conhecimentos compartilhados e o enquadramento da etnografia}

Downey e Ireland (apud Delli Zotti, 1996) apontam que as abordagens qualitativas ou quantitativas não são apropriadas ou inapropriadas, até que sejam aplicadas a um problema particular de pesquisa.

Feyerabend (2011), ao defender o pluralismo metodológico, critica a razão como faculdade pela qual os padrões da tradição racionalista se exercem, traduzindo-se em obediência a regras fixas e padrões imutáveis, estabelecendo-se e submetendo-se ao 'método' concentrado em aceitar apenas hipóteses que se ajustem a teorias confirmadas ou corroboradas e eliminar aquelas conjecturas que não se ajustem a fatos bem estabelecidos. De fato, não existem abordagens certas ou equivocadas, mas abordagens que melhor se ajustem a determinada proposta de pesquisa. Os métodos qualitativos são os mais adequados para a captação do verdadeiro comportamento social humano, promovendo maior envolvimento do pesquisador com o contexto investigado (Atkinson \& Hammersley, 1994).

Analisando os três tipos de pesquisa destacados por Creswell (2010), é possível afirmar que a pesquisa qualitativa é uma maneira de explorar e entender o significado que os indivíduos ou os grupos atribuem a um problema social ou humano, envolve variáveis subjetivas e o relatório final é escrito de forma mais flexível.

Taylor \& Bogdan (1997), por sua vez, também apontam algumas características do método qualitativo que o distinguem dos demais, a saber: as questões de pesquisa costumam ser mais abertas, uma vez que o pesquisador não prevê o que encontrará em campo; os sujeitos da pesquisa ou os contextos pesquisados não são reduzidos a variáveis, mas examinados por suas experiências vividas; os sujeitos são pesquisados em seu próprio referencial; não há uma verdade universalizável; o pesquisador deve suspender seu arcabouço axiológico para entrar em campo; não são descartados os outliners; existe preocupação ética com a repercussão da pesquisa nos objetos da pesquisa.

Revista de Gestão Social e Ambiental - RGSA, São Paulo, v. 11, n. 1, p. 38-55, jan./abr. 2017. 
Por meio da pesquisa qualitativa é possível estudar as partes para a compreensão do todo, porquanto o significado do conjunto resultante está incorporado na experiência vivida das pessoas objeto de estudo, sob a ótica das perspectivas interiores e percepções do próprio pesquisador (Merriam, 1998; Maxwell, 2008).

As pesquisas quantitativas, por outro lado, obedecem a um plano pré-estabelecido, a fim de enumerar ou mensurar eventos; utiliza teorias para elaborar hipóteses que procura comprovar; examina a relação de variáveis por métodos experimentais rigorosamente controlados; emprega, em geral, análise estatística (Creswell, 2010; Bryman, 2004; Hayati et al. 2006). Bryman (2004) ressalta que é comum que os contornos das principais etapas da pesquisa quantitativa sugiram que uma hipótese é deduzida a partir da teoria e, consequentemente, é testada. A redução a um conjunto acautelado de variáveis, rigidamente controladas pelo planejamento ou pela análise estatística, propicia medidas ou observações para a testagem de determinada teoria (Creswell, 2010).

Os métodos quantitativos, em razão, justamente, desta visão positivista, matemática, objetiva, tendem a fracassar na absorção da natureza genuína das práticas e dos comportamentos sociais humanos (Atkinson \& Hammersley, 1994).

$\mathrm{Na}$ linguagem quotidiana, as regras são relativas ao uso linguístico, às formas de viver e criar de cada lugar, as regras formais são reconstruídas a posteriori, sistemas, então, de descrição e não sistemas normativos (Grigoli \& Dal Lago, 1983). A etnografia apresenta-se como método apropriado ao estudo de uma cultura, sobretudo, por meio do cinema, uma vez que oferece técnicas de abordagem de aspectos da linguagem, das vestimentas, dos costumes e das representações de autoridade (Martinez, 1992; Hughes-Freeland, 1992).

Etnografia é um termo que advém do grego e refere-se à descrição de um grupo social (Chizzotti, 2014; Spradley, 1979). Um esforço intelectual para a obtenção de uma descrição densa (Geertz, 1973). Para Geertz (1973), a descrição consideraria diversas estruturas conceituais e simbólicas que conformam as ações humanas, não busca apenas a interpretação ou elucidação de fatos isolados, mas a interconexão entre os eventos e a construção de conjuntos a partir destes.

Apropriada pela antropologia, foi apresentada à comunidade científica como modo de descrição social de uma pessoa, da conformação cultural de uma população, ou descrição de cenários (Spradley \& Maccurdy, 1972; Chizotti, 2014). A acepção conhecida tem por marco o interacionismo simbólico (Schütz, 1962; Park \& Burgess, 1921; Blumer, 1937; Thomas, 1927; Chizotti, 2014). Para Geertz (1973), a etnografia é um esforço de comunicação entre culturas distintas. A abordagem etnográfica busca compreender o conhecimento internalizado nas pessoas pertencentes ao contexto examinado (Willis \& Trondman, 2000). Enquanto o propósito primeiro da etnografia é a imersão nas vivências e visões de mundo de um determinado grupo de pessoas, o etnógrafo permanece como observador externo do universo daqueles que estuda (Emerson et al., 1995).

A etnografia visa à captura daqueles conhecimentos incorporados nos sujeitos pertencentes a uma determinada comunidade, seus modos de pensar, viver e criar (Mattos e Castro, 2011; Willis \& Trondman, 2000; Bourdieu, 2001; Martinez, 1992). Esta metodologia foi utilizada em oportunidades pretéritas para estudar vida e morte da mulher bosquímana, métodos construtivos, tecelagem nativa, vestimentas das mulheres; afinidade linguística entre povos, técnicas de olaria, práticas religiosas, cremação de viúvas no sul da índia, bronzes antigos da Guiné, folclore (v.g. Almeida, 1963; Cinatti, 1963; Giese, 1963; Almeida \& Vieira, 1963; Dias, 1963; Carreira, 1963; Sousa, 1963; Mota, 1963; Lima, 1963), etc.

A abordagem etnográfica permitiu em investigações precedentes identificar a relação de determinada comunidade pesquisada com sua tecelagem, os grupos sociais envolvidos, o modo como o processo é realizado, o fato de que às mulheres era franqueado apenas encher bobinas, a fim de que o tecelão não interrompesse seu trabalho, etc. (Almeida \& Vieira, 1963). Isto demonstra que, de fato, a pesquisa etnográfica pode ser realizada para detalhar processos de compartilhamento de conhecimentos tácitos entre indivíduos (Ipe, 2003). As pessoas que transmitem os dados e as 
informações ao pesquisador são chamadas informantes. Para identificar os informantes dentro de uma população alvo, aplica-se uma metodologia chamada bola de neve (Atkinson \& Flint, 2001).

Os conhecimentos tradicionais, de modo geral, são transmitidos oralmente. A função da cultura nas sociedades tradicionais é construir e comunicar um plexo de conhecimentos (ReyesGarcia, 2010). Modernamente, a etnografia ainda é bastante útil como metodologia de condução dos estudos qualitativos, pois permite distinguir os elementos complexos e contraditórios da experiência cotidiana nas cidades (Hodges \& Denegri-Knott, 2012) e, consequentemente, nas organizações, inclusive, viabilizando o reconhecimento da influência das estruturas de conhecimento cultural nas percepções dos membros da organização (Johnston \& Everett, 2012).

A transferência de conhecimento é um fator de grande importância na construção e expansão da memória das organizações desde seus primórdios. Autores afirmam que esta transferência possui importância estratégica (Kaszás et al., 2016; Ùjhelyi, 2001). Nas organizações contemporâneas, pesquisas apontam que a cultura organizacional influencia a percepção das pessoas sobre a credibilidade do conhecimento e molda suas preferências para mecanismos específicos de transferência de conhecimento (Wei \& Miraglia, 2017). A cultura molda o comportamento (Norlim et al., 2003; Jiancheng, Lu \& Francesco, 2010), de modo que atitudes, normas subjetivas e controle comportamental influenciam pessoas a adotar posturas de troca de experiências (Razak et al., 2016). Para Jiancheng, Lu \& Francesco (2010), a motivação para compartilhamento e internalização de conhecimentos opera por meio do compromisso afetivo e as recompensas têm poucos efeitos diretos sobre as intenções finais, mas elas influenciarão indiretamente nas atitudes dos indivíduos.

Autores defendem que o comportamento de compartilhamento de conhecimento não é influenciado pelos valores organizacionais em si, mas pelo grau de sua internalização pelos membros da organização (Michailova \& Minbaeva, 2012). Em culturas individualistas, que evitam a incerteza e não costumam manter relações estreitas no local de trabalho, há nítida preferência pela instrução formal, fornecida fora do local de trabalho ao invés da transferência de conhecimentos pela troca de experiências (Kaszás et al., 2016). Atualmente, estudos na área de tecnologias da informação buscam encontrar modelagens adequadas para otimizar o compartilhamento de conhecimentos nas organizações e indicadores de cooperação (v.g. Jakobsone et al., 2017).

Não apenas internamente, mas também entre organizações ocorre transferência de conhecimentos. Os canais mais comuns operam no nível da atividade e incluem imitação, demonstração, observação, colaboração entre empresas e troca de documentos (Werner et al., 2015).

O pressuposto fundamental da etnografia é a interação direta com pessoas em suas vivências quotidianas, uma vez que isto viabilizaria melhor compreensão de suas concepções, práticas, motivações, comportamentos, procedimentos, bem como o significado que atribuem a suas ações (Chizotti, 2014). A entrevista semiestruturada, muito comum, é uma técnica de coleta de dados que pressupõe a conversação continuada entre participante e pesquisador, dirigida de acordo com os objetivos da pesquisa (Queiroz, 1998).

São características básicas da pesquisa etnográfica, a observação participante, o estudo de campo, estudo de pequenos grupos relativamente homogêneos e limitados geograficamente, bem como a descrição interpretativa da cultura (Wilson, 1997; Ezpeleta \& Rockwell, 1989; Bogdan \& Birklen, 1994; Beaud \& Weber, 1998; Goetz \& Lecompte, 1984; Wolcott, 1994; Chizotti, 2014).

\section{MÉTODO}

Realizou-se uma revisão integrativa da literatura para selecionar uma amostra representativa do material publicado sobre etnografia e sustentabilidade, a fim de levantar evidências científicas. Por meio da revisão de literatura, o pesquisador mapeia o território intelectual já existente (Tranfield et al., 2003). As etapas do processo de revisão são planejadas e 
executadas explicitamente, tendo a síntese por ápice, materializada no relatório (Crossan \& Apaydin, 2010).

Foram definidos os eixos de pesquisa e as palavras-chave para cada eixo, com ênfase em sustentabilidade, cultura organizacional e etnografia. As bases de dados utilizadas foram Scopus, Science Direct e Web of Science.

Nas bases, a pesquisa se restringiu a periódicos, excluindo-se anais de eventos e capítulos de livros. Os artigos foram filtrados pelos títulos, resumos e palavras-chave. Foi utilizada a seguinte expressão de busca: ("sustainability" or "sustainable" or "environmental" or "environment") and ("organizational culture" or "culture" or "knowledge sharing" or "knowledge transfer" or "organizational values") and ("ethnography" or "method" or "methodology" or "ethnographic"). Considerando o espectro interdisciplinar de abrangência dos termos, deu-se maior ênfase à área das ciências sociais aplicadas. Como categorias de análise, foram consideradas as seguintes: sustentabilidade, cultura e cultura organizacional, compartilhamento de conhecimentos, etnografia e método qualitativo.

Os valores e as preferências do pesquisador foram determinantes na escolha e interpretação dos artigos componentes do portfólio analisado.

Foram selecionados 56 artigos, sem delimitação temporal. Os periódicos que mais se destacaram em termos da temática da sustentabilidade foram o Journal of Cleaner Production, Procedia - Social and Behavioral Sciences e International Journal of Sustainability in Higher Education. Os artigos considerados na análise dividiram-se nos seguintes anos:

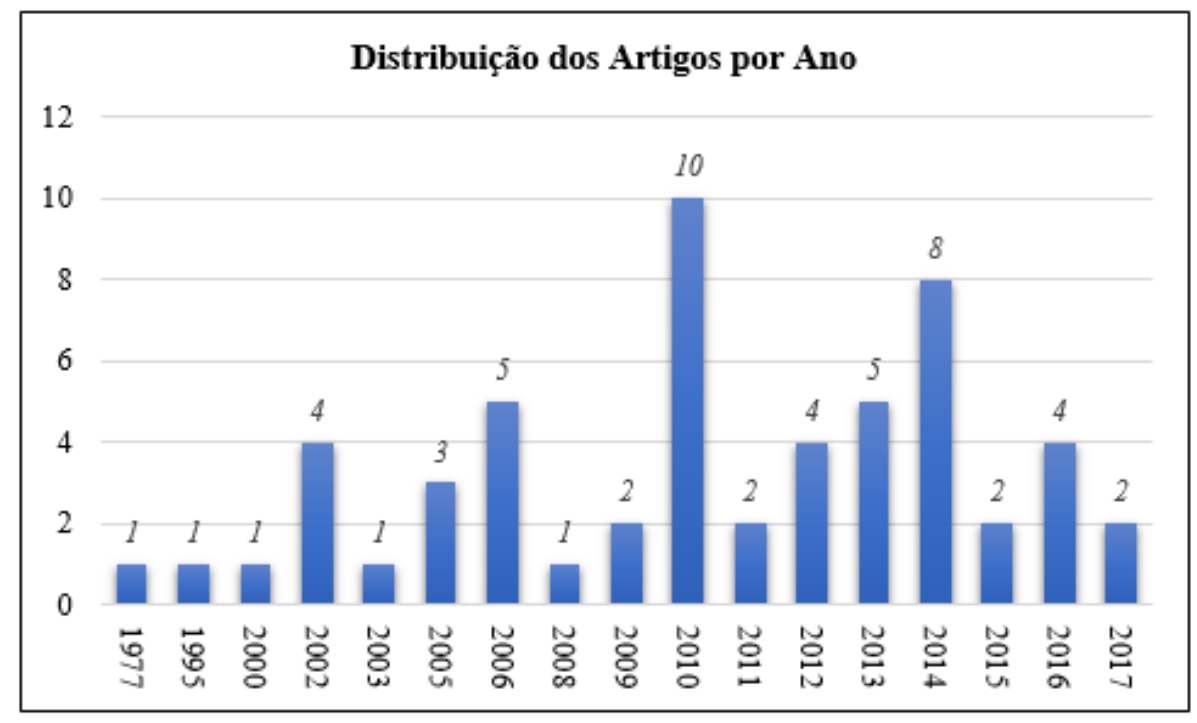

Figura 1 - Distribuição temporal dos artigos selecionados

Fonte: Dados da pesquisa (2017)

Para complemento desta pesquisa, foi necessária a realização de pesquisa bibliográfica específica sobre a temática da etnografia e etnometodologia. A pesquisa bibliográfica abrangeu a leitura, análise e interpretação de livros e periódicos, acompanhada de compilações de excertos por meio de fichamento.

Esta pesquisa possui caráter exploratório e qualitativo. A abordagem desta pesquisa é qualitativa, uma vez que os resultados obtidos estão atrelados às escolhas e valores do pesquisador ao determinar seu escopo. A pesquisa qualitativa é uma maneira de explorar e entender o significado que os indivíduos ou os grupos atribuem a um problema social ou humano (Creswell, 2010).

De acordo com Triviños (1987), estudos exploratórios servem para revolver os problemas de pesquisa, trazer luz a fenômenos pouco estudados e até mesmo servir de precedentes para estudos descritivos e experimentais. 
Não se trata de um estudo bibliométrico. Com base nos achados da literatura, esse estudo sistematiza um protocolo etnográfico para investigar a cultura organizacional voltada à sustentabilidade e não pretende realizar a investigação, mas propor diretrizes para estudos empíricos futuros.

\section{APRESENTAÇÃO DOS RESULTADOS}

As preocupações com a sustentabilidade adquiriram relevo com o decurso do tempo em razão de importantes impactos que eventos, passados e atuais, têm o condão de acarretar às gerações futuras, razão pela qual vêm sendo incorporadas à rotina das organizações.

A cultura organizacional baseia-se no conjunto de valores compartilhado por todos os membros da organização, que guarda conexão intrínseca com as idiossincrasias das pessoas em seu meio social (Çakar \& Ertürk, 2010). As influências externas, por outro lado, tendem a influenciar na cognição interna, comportamento e nos valores particulares de um indivíduo (Malhotra \& Galletta, 2005). Neste sentido, a cultura organizacional pode ser compreendida segundo o modelo a seguir:

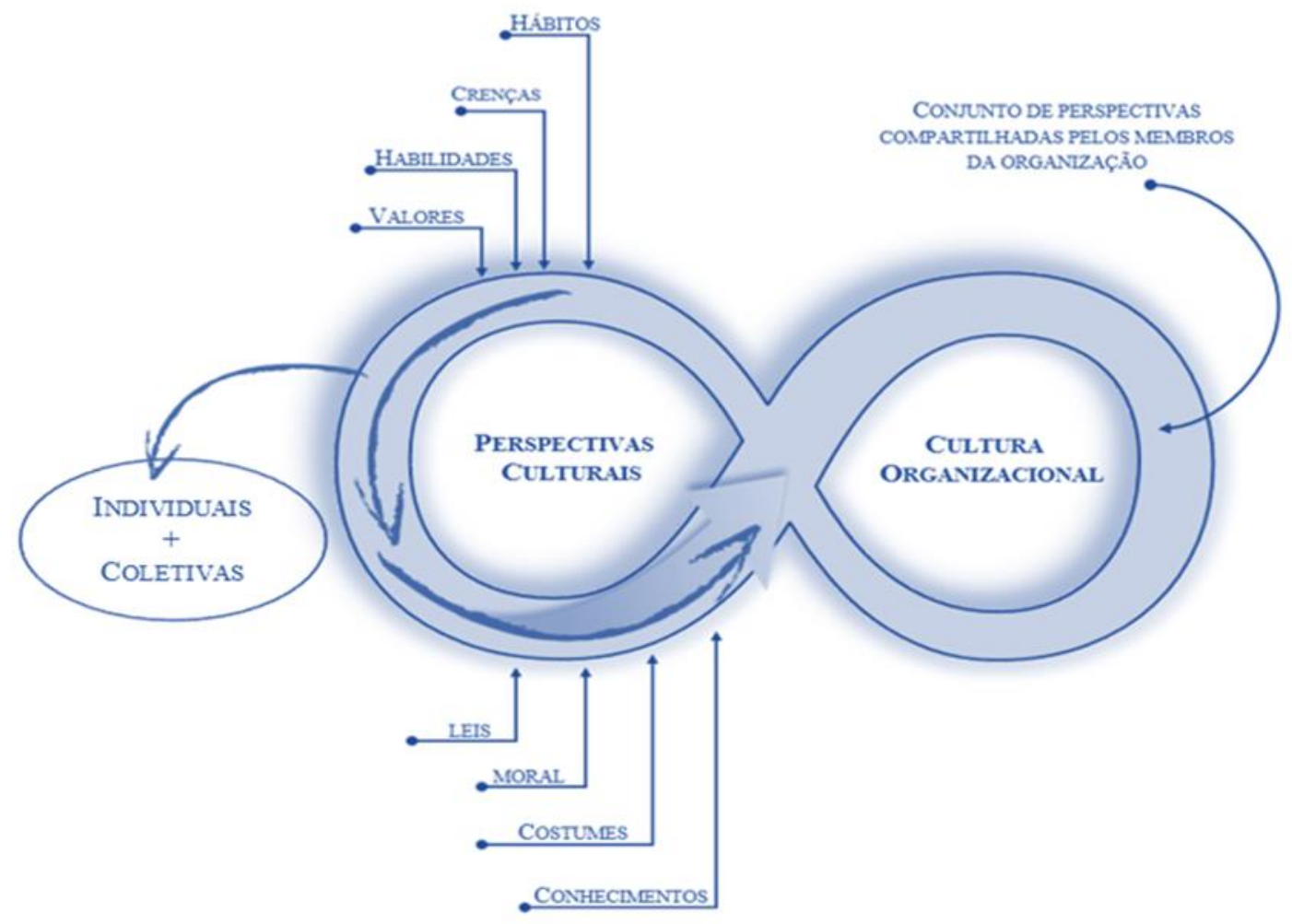

Figura 2 - Influências sobre a cultura organizacional

Fonte: Elaborado pelos autores (2016) a partir dos dados da pesquisa

Etnografia é um método adequado ao estudo da cultura da sustentabilidade nas organizações, uma vez que permite identificar o estado anímico dos indivíduos envolvidos no processo de transformação organizacional, seus valores e suas crenças. Boas práticas sustentáveis estão associadas à cultura organizacional, muitas vezes demandam processos de ruptura e aprendizagem para que sejam instituídas e institucionalizadas (Maon et al, 2009; Senge, 1990).

Inovações e incentivos sociais são fatores críticos na transformação rumo a práticas mais sustentáveis (Tukker \& Jansen, 2006; Petala et. al., 2010; Hallstedt et al, 2013), razão pela qual a investigação da cultura organizacional voltada à sustentabilidade é também uma cultura aberta à inovação (Vidal-Salazar et al., 2012) e aceitação de mudanças nas rotinas existentes. 
A abordagem etnográfica, ao buscar a compreensão do conhecimento internalizado nas pessoas pertencentes ao contexto sob exame (Willis \& Trondman, 2000), requer o contato próximo com os informantes do estudo e uma capacidade grande de observação e análise crítica por parte do pesquisador.

Para detalhar processos de compartilhamento entre indivíduos, próprios da criação e perpetuação de aspectos culturais, de conhecimentos tácitos, ou seja, adquirido ao longo da vida pela experiência (Nonaka \& Takeuchi, 1997), é útil a abordagem etnográfica (Ipe, 2003). O compartilhamento tácito de conhecimentos, de modo geral, ocorre de forma mais natural. $\mathrm{O}$ conhecimento explícito possui processos mais complexos de compartilhamento (Zhigang Li et al., 2016). Como o conhecimento dos membros de uma organização é transmitido, sobretudo, oralmente (Reyes-Garcia, 2010), por meio da linguagem adotada por aquela comunidade (Grigoli \& Dal Lago, 1983), a forma mais adequada de coletar dados para abordar, etnograficamente, um tema é por meio de entrevistas (Queiroz, 1998). Além disso, a observação participante, consistente na convivência rotineira com o contexto e os sujeitos pesquisados, viabiliza a interação com as vivências do ambiente investigado, possibilitando melhor compreensão de sua forma de pensar (Chizotti, 2014).

Questão central da pesquisa em ciências sociais é o desenvolvimento de inferências descritivas e causais a partir de dados observáveis (King, Keohane, \& Verba, 1994). Partindo-se desta premissa, sugere-se a etnografia como metodologia para desenvolver inferências descritivas acerca da relação entre as práticas de sustentabilidade adotadas e a cultura de uma organização.

A partir da revisão da literatura elaborada, é possível propor um modelo de processo para elaboração do estudo etnográfico da cultura organizacional voltada à sustentabilidade: um protocolo etnográfico para a investigação da cultura da sustentabilidade nas organizações. É necessário ter cuidado com a abordagem do tema, evitando induzir os informantes que expressem não suas reais práticas, mas aquilo que acreditam ser mais adequado.

Para fins de aplicação desta metodologia, recomenda-se o estabelecimento de premissas epistemológicas, a primeira delas de que a cultura organizacional se constitui de um sistema de conhecimento compartilhado entre os indivíduos e transmitido socialmente ao longo do tempo entre os membros da organização (Everett \& Johnston, 2012). A segunda premissa é a de considerar a cultura como ambiente das relações interpessoais. No caso da cultura orientada à sustentabilidade, é de se ver que, nas organizações, esta deve fazer parte da estratégia corporativa, todavia, é dependente do comportamento e da cooperação dos indivíduos.

O etnógrafo procura identificar o significado das relações sociais (Mattos e Castro, 2011). O estudo etnográfico enfatiza a proposta de pesquisa e com esta preocupa-se, muito mais do que com o procedimento, em si, de coleta de dados, que exige contato direto com os sujeitos da pesquisa (Chizotti, 2014; Taylor \& Bogdan, 1997).

Como em qualquer pesquisa qualitativa, é imprescindível que a proposta de pesquisa justifique as escolhas do pesquisador, evidenciando seu perfil e seu papel - conexões passadas com o local, forma de obter acesso a informações sensíveis, etc. (Creswell, 2010).

A prática da pesquisa etnográfica envolve muitas tarefas com as quais os pesquisadores mais novos não estão familiarizados. Sem a pretensão de criar um processo universalmente aceito, com base em Chizotti (2014), Spradley (1979) e Creswel (2010), formulou-se o fluxo abaixo para orientação de futuras pesquisas: 
Etnografia como estratégia investigativa da cultura organizacional para a sustentabilidade.

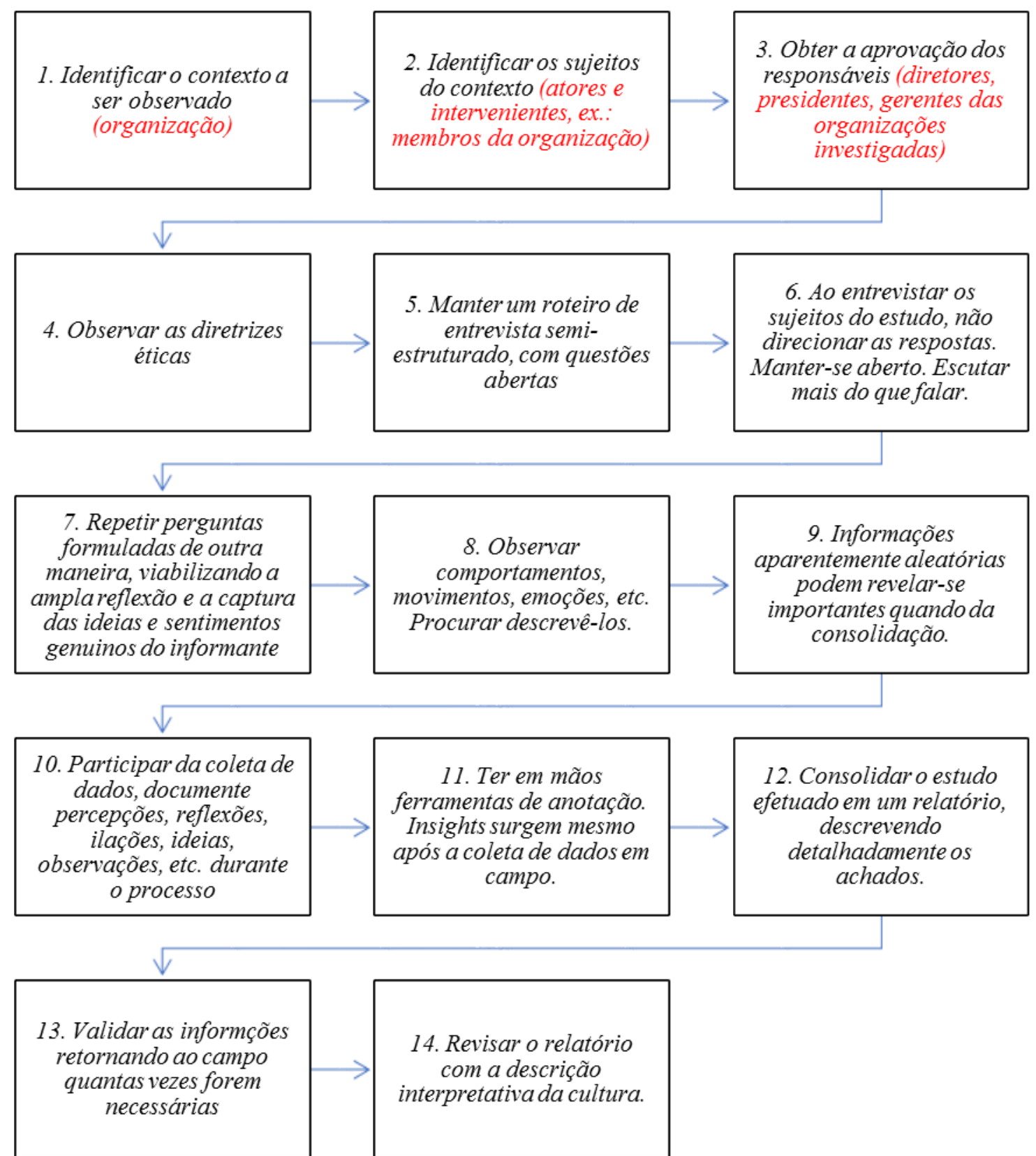

Figura 3 - Protocolo etnográfico para investigação da cultura da sustentabilidade Fonte: Elaborado pelo autor a partir dos dados da pesquisa (2016)

O protocolo etnográfico sugerido é apenas um guia para a investigação da cultura organizacional, não pretende, como dito, ser um modelo universal. Como se sabe, a grande contribuição da pesquisa qualitativa não diz respeito à extensão pura e simples dos resultados obtidos para contextos análogos, mas sim na construção de uma teoria que poderá orientar a elaboração de outros estudos (Maxwell, 2008; Creswell, 2010).

Creswell (2010) atenta para as questões éticas que circundam o método qualitativo, de forma mais intensa do que nas abordagens quantitativas - uma vez que há necessariamente interação direta com os objetos da pesquisa -, e que merecem atenção do pesquisador, o qual se encontra envolvido no contexto pesquisado e relaciona-se intensamente com os participantes do estudo.

O pesquisador, ao entrar em campo, introduz-se, de certa forma, no ambiente pesquisado (Chizotti, 2014). Por meio da imersão nas vivências e visões de mundo de um determinado grupo de pessoas, o etnógrafo permanece como observador externo do universo daqueles que estuda (Emerson et al., 1995), uma das razões pelas quais a pesquisa etnográfica é sugerida como 
estratégia investigativa da cultura de sustentabilidade, permitindo observar as práticas e políticas do contexto pesquisado. Ao participar e observar o contexto e o comportamento dos sujeitos da pesquisa neste ambiente durante certo tempo, de forma indutiva, o pesquisador tem a oportunidade de analisar condutas que dificilmente seriam referidas abertamente pela comunidade sob estudo (Malinowski, 1984), daí a importância de estar atento às reações, ouvir muito e documentar as observações.

O texto etnográfico resultante da pesquisa deve buscar uma descrição refinada das observações, chamada descrição densa (Geertz, 1973). O pesquisador deve apresentar uma perspectiva holística do fenômeno em estudo, de seu ambiente, de seus atores (Chizotti, 2014). Para que este relatório seja possível, é imprescindível que as inferências, reflexões e os sentimentos do próprio pesquisador com relação ao campo de pesquisa sejam documentados no curso das investigações.

Os significados e as perspectivas objetivadas pelo pesquisador, muitas vezes, não são considerados importantes pelos informantes, razão pela qual a estrutura das entrevistas, apesar de aberta e passível de alterações no curso do processo, deve contar com o dirigismo do pesquisador.

As etnografias, por sua natureza, privilegiam estudos de pequenos grupos ou de comunidades localizadas em um determinado ambiente, razão pela qual se adequam aos estudos organizacionais, com a vantagem de permitir a reconstrução de cenários e a descrição de práticas (Spradley \& Maccurdy, 1972). A força do imperativo etnográfico no desenho da pesquisa qualitativa é refletida pelo grau de congruência entre as inferências descritivas extraídas dos dados etnográficos e o contexto teórico em que estas deduções estão situadas (Everett \& Johnston, 2012).

\section{CONSIDERAÇÕES FINAIS}

A sustentabilidade é um tema relevante na atualidade, sobretudo, em razão dos diversos reflexos econômicos que as vicissitudes socioambientais acarretam em nível global. Adotar posturas sustentáveis dentro das organizações pressupõe uma mudança cultural, a fim de que os processos internos sejam adaptados visando à promoção do bem-estar da coletividade e, ainda, que o desenvolvimento sustentável integre a estratégia corporativa e contribua para o crescimento da organização.

O papel das instituições de ensino é considerado importante na formação de profissionais mais conscientes e atentos às demandas da sustentabilidade.

Na pesquisa etnográfica, considera-se a especificidade das ações, as emoções, perspectivas e os significados dos sujeitos envolvidos, bem como as características do contexto pesquisado. $\mathrm{O}$ trabalho em campo envolve métodos indutivos. As categorias, conjecturas e problemáticas a pesquisar dificilmente poderão ser definidas previamente, de forma hermética. $\mathrm{O}$ caráter qualitativo da pesquisa reflete-se na flexibilização do plano de estudo.

A imersão e o envolvimento direto do pesquisador em campo permitem que sejam levantados dados e informações que não seriam compartilhados de outra forma.

A etnografia figura-se adequada para a investigação de uma cultura organizacional voltada à sustentabilidade, uma vez que, por sua própria natureza, permite uma descrição densa dos ambientes, dos atores e das práticas, bem como das relações dos sujeitos com suas ações e o sentimento que lhes proporciona. Pela caracterização de uma cultura organizacional voltada à sustentabilidade, por meio da etnografia, será possível mapear os processos necessários à transformação organizacional em prol de práticas mais sustentáveis.

O grande contributo desta pesquisa consiste na caracterização de uma metodologia adequada à investigação do fenômeno 'sustentabilidade' nas organizações, a partir da exploração de aspectos culturais, servindo de diretriz para futuros estudos. 


\section{REFERÊNCIAS}

Adomßent, M., Fischer, D., Godemann, J., Herzig, C., Otte, I., Rieckmann, M., Timm, J. (2014) Emerging areas in research on higher education for sustainable development e management education, sustainable consumption and perspectives from Central and Eastern Europe. Journal of Cleaner Production, Elsevier Ltd. (62), 1-7.

Aidar, M. M., Brisola, A. B., Motta, F. C. P., Wood Jr., T. (2002) Cultura organizacional brasileira.

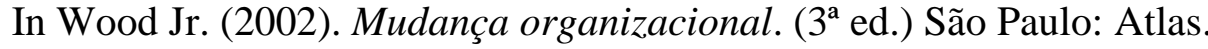

Almeida, M.E.C. (1963) Da vida e da morte da mulher bosquímana (Angola). In Actas do Congresso Internacional de Etnografia. Promovido pela Câmara Municipal de Santo Tirso de 10 a 18 de julho de 1963. Colóquio de Etnografia Comparada, 4. Porto: Imprensa Portuguesa.

Almeida, M. E.. de C., Vieira, M. (1963) Breves notas sobre a tecelagem nativa na Guiné Portuguesa. In Actas do Congresso Internacional de Etnografia. Promovido pela Câmara Municipal de Santo Tirso de 10 a 18 de julho de 1963. Colóquio de Etnografia Comparada. 4. Porto: Imprensa Portuguesa.

Ammenberg, J., Sundin, E. (2005) Products in environmental management systems: drivers, barriers and experiences. Journal of Cleaner Production, 13(4), 405-415.

Atkinson, P., Hammersley, M. (1994) Ethnography and participant observation. Handbook of qualitative research, 1(23), 248-261.

Atkinson, R., \& Flint, J. (2001) Accessing hidden and hard-to-reach populations: snowball research strategies. Social Research Update, 33(1), 1-4.

Aussenac-Gilles, N., Gandon, F. (2013) From the knowledge acquisition bottleneck to the knowledge acquisition overflow: a brief French history of knowledge acquisition. International Journal of Human-Computer Studies, 71(2), February, 157-165.

Barba-Sanchez, V., Atienza-Sahuquillo, C. (2010) Integration of the environment in managerial strategy: application of the resource-based theory of competitive advantage, dynamic capabilities and corporate social responsibilities. African Journal of Business Management, 4 (6).

Banerjee, S. B. (2002) Corporate environmentalism. The construct and its measurement. Journal of Business Research, 55, 177-191.

Beaud, S., Weber, F. (1998) Guide de l' enquete de terrain: Produire el analyser des donnés ethnographiques. Paris: La Decouverte.

Benjamins, V. R. (2013) Information is not knowledge, knowledge is not wisdom, wisdom is not truth.. International Journal of Human-Computer Studies, 71(2), February, 166-170.

Benn, S., Kearins, K. (2012) Sustainability and organizational change. In D. Boje, B. Burnes \& J. Hassard (Eds.) Handbook of organizational change, 535-551. London, England: Sage.

Benn, S. Edwards, M. Angus-Leppan, T. (2013) Organizational learning and the sustainability community of practice: the role of boundary objects. Organization \& Environment, 26(2), 184-202.

Beske, P. (2012) Dynamic capabilities and sustainable supply chain management. Int Jnl Phys Dist \& Log Manage, 42, 372-387.

Blumer, H. (1937) Symbolic Interacionism. Perspective and Method, Chicago, University of Chicago Press. 
Bogdan, R., Biklen, S. (1994) Investigação qualitativa em educação: uma introdução à teoria e aos métodos. Porto: Porto Ed.

Bourdieu, P. (2001) Meditações pascalianas. Rio de Janeiro: Bertrand.

Burke, L. Longsdon, J. M. (1996) How corporate social responsibility pays off. Long Range Planning, Elsevier Science Ltd, 29(4), 495 to 502. Great Britain.

Bryman, A. (2004) Social research methods. (2. ed.) Oxford: Oxford University Press, 140-162. Cap. 6 - The nature of quantitative research.

Çakar, N. D., Ertürk, A. (2010) Comparing innovation capability of small and medium-sized enterprises: examining the effects of organizational culture and empowerment. Journal of Small Business Management, 48(3), 325-359.

Carreira, A. (1963) Aspectos da influência da cultura portuguesa na área compreendida entre o rio Senegal e o norte da Serra Leoa. In Actas do Congresso Internacional de Etnografia. Promovido pela Câmara Municipal de Santo Tirso de 10 a 18 de julho de 1963. Colóquio de Etnografia Comparada. 4. Porto: Imprensa Portuguesa.

Castro, C. M. (1977) A prática da pesquisa. São Paulo: McGraw-Hill do Brasil.

Cinatti, R.(1963) Tipos de casas timorenses e um rito de consagração. In Actas do Congresso Internacional de Etnografia. Promovido pela Câmara Municipal de Santo Tirso de 10 a 18 de julho de 1963. Colóquio de Etnografia Comparada, 4. Porto: Imprensa Portuguesa.

Chizotti, A. (2014) Pesquisa qualitativa em ciências humanas e sociais. ( $6^{\mathrm{a}}$ ed.) Petrópolis-RJ: Vozes.

Creswell, J. W. (2010) Projeto de pesquisa: métodos qualitativo, quantitativo e misto. (3. ed.) Porto Alegre: Artmed, 177-205.

Crossan, M. M., Apaydin, M. (2010) A multi-dimensional framework of organizational innovation: a systematic review of the literature. Journal of Management Studies, 47(6), 1154-1191.

Delli Zotti, G. (1996) Quale quantitá e quanta qualitá nella ricerca sociale: tra integrazione e convergenza, 136-166. In C Cipolla \& A De Lillo (orgs.). Il Sociologo e le Sirene: la Sfida dei Metodi Qualitativi. Angeli, Milão.

Dalkir, K. (2005) Knowledge management in theory and practice. Boston: Elsevier.

Dicle, U., Köse, C. (2014) The impact of organizational learning on corporate sustainability and strategy formulation with the moderating effect of industry type. 10th International Strategic Management Conference. Procedia - Social and Behavioral Sciences 150, 958 - 967.

Emerson, R. M., Fretz, R. I., Shaw, L. L. (1995) Writing ethnographic fieldnotes. The University of Chicago Press, Chicago (USA).

Erturgut, R., Soysekerci, S. (2009) The problem of sustainability of organizational success in public educational institutions: a research on the education administrators in Turkey. World Conference on Educational Sciences 2009. Procedia Social and Behavioral Sciences, (1), 2092-2102.

Everett, J. L., \& Johnston, K. A. (2012) Toward an ethnographic imperative in public relations research. Public Relations Review, 38(4), 522-528.

Ezpeleta, J., Rockwell, E. (1989) Pesquisa participante. (2ª ed.) São Paulo: Cortez.

Feyerabend, P. (2011) A ciência em uma sociedade livre. (1 ${ }^{\mathrm{a}}$ ed.) São Paulo: Editora Unesp. 
Gaines, B. R. (2013). Knowledge acquisition: Past, present and future. International Journal of Human-Computer Studies, 71(2), February, 135-156.

Geertz, C. (1973) The interpretation of cultures. New York: Basic Books.

Giddings, B., Hopwood, B., O’brien, G. (2002) Environment, economy and society: fitting them together into sustainable development. Sustainable Development, 10(4), 187-196.

Giese, W. (1963) Os capotes das mulheres açorianas. In Actas do Congresso Internacional de Etnografia. Promovido pela Câmara Municipal de Santo Tirso de 10 a 18 de julho de 1963. Colóquio de Etnografia Comparada, 4. Porto: Imprensa Portuguesa.

Grigoli, P. P. e Dal Lago, A. (1983) Etnometodologia / a cura di Pier Paolo Giglioli e Alessandro Dal Lago. Bologna : Il mulino.

Godemann, J., Haertle, J., Herzig, C., Moon, J. (2014) United Nations supported Principles for Responsible Management Education: purpose, progress and prospects. Journal of Cleaner Production, Elsevier Ltd. (62), 16-23.

Goetz, J.P., Lecompte, M. D. (1984) Ethnography: principles and practice. San Diego, CA: Academic Press.

Gomes, D.V. (2010) A solidariedade social e a cidadania na efetivação do direito a um meio ambiente ecologicamente equilibrado. Direito e Liberdade, 3(2), 203-214.

Hallstedt, S.I., Thompson, A.W., Lindahl, P. (2013) Key elements for implementing a strategic sustainability perspective in the product innovation process. Journal of Cleaner Production, 51, 277-288.

Harris, L. C., Crane, A. (2002) The greening of organizational culture: Management views on the depths, degree and diffusion of change. Journal of Organizational Change Management, 15(3), 214-234.

Hayati, D., Karami, E. \& Slee, B. (2006) Combining qualitative and quantitative methods in the measurement of rural poverty. Social Indicators Research, 75, 361-394, springer.

Hesselbarth, C., Schaltegger, S. (2014) Educating change agents for sustainability - learnings from the first sustainability management master of business administration. Lüneburg, Germany. Journal of Cleaner Production, (62), 24-36.

Hodges, C. E., \& Denegri-Knott, J. (2012) Transforming the city: the potential for urban ethnographies of PR: The case of Latin America. Public Relations Review, 38(4), 529-540.

Hughes-Freeland, F. (1992) Representation by the other: Indonesian cultural documentation. In Crawford, P. I. e Turton, D. (Org.) Film as Etnography. New York: Manchester University Press.

Hussein, N., Mohamad, A., Noordin, F., Ishak, N.A. (2014) Learning organization and its effect on organizational performance and organizational innovativeness: a proposed framework for malaysian public institutions of higher education. Procedia - Social and Behavioral Sciences, (130), $299-304$.

James, L. R., Choi, C. C., Ko, C. H. E., McNeil, P. K., Minton, M. K., Wright, M. A., \& Kim, K. I. (2008) Organizational and psychological climate: a review of theory and research. European Journal of Work and Organizational Psychology, 17(1), 5-32.

Jakobsone, A., Cakula, S., \& Florea, M. (2017) Modelling of knowledge sharing for the provision of sustainable cooperation between adult educational institutions and enterprises. Procedia Computer Science, 104, 160-165. 
Johnston, K. A., \& Everett, J. L. (2012) Employee perceptions of reputation: an ethnographic study. Public Relations Review, 38(4), 541-554.

Kahn, J. S. (1975) Introdución. In: Goodenaugh, W. H., Kahn, J.S., Malinowski, A. L., Bronislaw, T. Y. L. O. R., Edward, B. W., \& Leslie, A. (1975) El concepto de cultura: textos fundamentales. Barcelona: Anagrama.

Kaszás, N., Keller, K., \& Birkner, Z. (2016) The role of transferring knowledge in case of nonbusiness sector projects. Procedia-social and behavioral sciences, 221, 226-235.

King, G., Keohane, R. O., \& Verba, S. (1994) Designing social inquiry: scientific inference in qualitative research. Princeton university press.

Leonidou, L.C., Leonidou, C.N., Fotiadis, T.A., Aykol, B. (2015) Dynamic capabilities driving an eco-based advantage and performance in global hotel chains: the moderating effect of international strategy. Tourism Management, 50, 268-280.

Liboni LB, Jabbour CJC, Jabbour A, Devika K. (2016 - In press) Sustainability as a dynamic organizational capability: a systematic review and a future agenda toward a sustainable transition. Journal of Cleaner Production.

Lima, F. de C.P. de. (1963) Contribuição para o estudo do folclore de Moçambique. In Actas do Congresso Internacional de Etnografia. Promovido pela Câmara Municipal de Santo Tirso de 10 a 18 de julho de 1963. Colóquio de Etnografia Comparada, 4. Porto: Imprensa Portuguesa.

Linnenluecke, M. K., Griffiths, A. (2010) Corporate sustainability and organizational culture. Journal of World Business (45), 357-366.

Lozano, R. (2011) Creativity and organizational learning as means to foster sustainability. Sustainable Development. John Wiley \& Sons, Ltd.

Malinowski, B. (1984) Argonauts of the western pacific. prospect highs: Waveland Press.

Malhotra, Y., \& Galletta, D. (2005) A multidimensional commitment model of volitional systems adoption and usage behavior. Journal of Management Information Systems, 22(1), 117-151.

Maon, F., Lindgreen, A., Swaen, V. (2009) Designing and implementing corporate social responsibility: an integrative framework grounded in theory and practice. Journal of Business Ethics, 87(1), 71-89.

Martinez, W. (1992) Who constructs anthropological knowledge? Towards a theory of ethnographic film spectatorship. In: Crawford, P. I. e Turton, D. (Org.) Film as Etnography. New York: Manchester University Press.

Mattos, C.L.G., Castro, P. A. (Org.). (2011). Etnografia e educação: conceitos e usos. Campina Grande: Eduepb.

Maxwell, J. A. (2008) Designing a qualitative study. In: Bickman, L., Rog, D. (Ed.) Handbook of applied social research methods. Thousand Oks CA: Sage, 214-253.

Merad, M., Derchi, N., Marcel, F. (2014) A pragmatic way of achieving highly sustainable organisation: governance and organisational learning in action in the public french sector. Safety Science, Elsevier Ltd.(69), 18-28.

Merriam, S. B. (1998) Qualitative research and case study applications in education. San Francisco -CA: Jossey-Bass. 
Michailova, S., \& Minbaeva, D. B. (2012) Organizational values and knowledge sharing in multinational corporations: The Danisco case. International Business Review, 21(1), 59-70.

Moore, J. (2005) Seven recommendations for creating sustainability education at the university level: a guide for change agents. International Journal of Sustainability in Higher Education, 6(4), 326-339.

Morel, C.M. et al. (2004) A pesquisa em saúde e os objetivos do milênio: desafios e oportunidades globais, soluções e políticas nacionais. Ciência \& saúde coletiva, 9(2), 261-270.

Mota, A. T. da. (1963). Bronzes antigos da Guiné. In: Actas do Congresso Internacional de Etnografia. Promovido pela Câmara Municipal de Santo Tirso de 10 a 18 de julho de 1963. Colóquio de Etnografia Comparada, 4. Porto: Imprensa Portuguesa.

Murphy, P.; R. Poist. (1995) Role of relevance of logistics corporate environmentalism: an empirical assessment. International Journal of Physical Distribution \& Logistics Management 25 (2), 5-19.

Nicolaides, A. (2006) The implementation of environmental management towards sustainable universities and education for sustainable development as an ethical imperative. International Journal of Sustainability in Higher Education, 7(4), 414-424.

Nonaka, I., \& Takeuchi, H. (1997) Criação de conhecimento na empresa: como as empresas japonesas geram a dinâmica da inovação. Rio de Janeiro: Campus, 16, 360.

Oecd - Organisation for Economic Co-Operation and Development. (2008) Promoting Sustainable Consumption. Good Practices in Oecd Countries. Recuperado em 25 mar. 2016, de: http://www.oecd.org/dataoecd/1/59/40317373.pdf. Paris..

Programa das Nações Unidas para o Desenvolvimento - Pnud. Organização das Nações Unidas ONU. (2015). Relatório de Desenvolvimento Humano 2015: O Trabalho como Motor do Desenvolvimento Humano. Recuperado em: 21 abril 2016, de: http://www.pnud.org.br/HDR/arquivos/RDHglobais/hdr2015_ptBR.pdf.

Park, R. E., Burgess. E. W. (1921) Introduction to the sciences of sociology, Chicago: University of Chicago Press.

Petala, E., Wever, R., Dutilh, C., Brezet, H. (2010) The role of new product development briefs in implementing sustainability: a case study. Journal of Engineering and Technology Management 27 (3e4), 172e182.

Peters, N.J., Hofstetter, J.S., Hoffmann, V.H. (2011) Institutional entrepreneurship capabilities for inter-organizational sustainable supply chain strategies. Int Jrnl Logistics Management 22, 52-86.

Porter, M. E., Kramer, M. R. (2011) Creating shared value. Harvard Business Review, 89(1/2), $62-$ 77, 16, Jan/Feb.

Queiroz, M. I. P. (1988) Relatos orais: do "indizível" ao "dizível". In: Von Simson, O. M. (org. e intr.). Experimentos com histórias de vida (Itália-Brasil). São Paulo: Vértice, Editora Revista dos Tribunais, Enciclopédia Aberta de Ciências Sociais, 5, 68-80.

Razak, N. A., Pangil, F., Zin, M. L. M., Yunus, N. A. M., \& Asnawi, N. H. (2016) Theories of knowledge sharing behavior in business strategy. Procedia Economics and Finance, 37, 545-553.

Reyes-Garcia, V. (2010) The relevance of traditional knowledge systems for ethno pharmacological research: theoretical and methodological contributions. Journal of Ethnobiology and

Ethnomedicine, 6, 32. 
Reuter, C., Foerstl, K., Hartmann, E., Blome, C. (2010) Sustainable Global Supplier Management: the role of Dynamic Capabilities in achieving competitive advantage. Journal of Supply Chain Management, 45-63.

Russo, M.V. (2003) The emergence of sustainable industries: building natural capital. Strategic Management Journal, 24 (4), 317-331.

Schrettle, S. Hinz, A., Rathje, M.S., Friedli, T. (2014) Turning sustainability into action: Explaining firm's sustainability efforts and their impact on firm performance. Intern. Journal of Production Economics 147, 73-84.

Senge, P. M. (1990) The fifth discipline: the art and practice of learning organization. New York Doubleday.

Shriberg, M. (2002) Toward sustainable management: the University of Michigan Housing Division's approach. Journal of Cleaner Production, 10(1), 41-45.

Sitarz, D. (1993). Agenda 21: The earth summit strategy to save our planet.

Sneddon, C., Howarth, R.B., Norgaard, R. B. (2006) Sustainable development in a post-Brundtland world. Ecological economics, 57(2), 253-268.

Spangenberg, J.H., Alastair, F.-L., Blincoe, K. (2010) Design for Sustainability (DfS): the interface of sustainable production and consumption. Journal of Cleaner Production, 18 (15), 1485-1493.

Spradley, J.P., Maccurdy, D.W. (Org.) (1972) The cultural experience: ethnography in complex society. Chicago: Science Research Associates.

Spradley, J.P. (1979) The ethnographic interview. New York: Holt/Rinehart/Winston.

Sousa, E.T. De. (1963) A cremação de viúvas no Sul da Índia. In: Actas do Congresso Internacional de Etnografia. Promovido pela Câmara Municipal de Santo Tirso de 10 a 18 de julho de 1963.

Colóquio de Etnografia Comparada, 4. Porto: Imprensa Portuguesa.

Schütz, A. (1962) The problem of social reality. Collected papers 1. The Hague. Martinus Nijhoff.

Taylor, S. J., Bogdan, R. (1997) Introduction to qualitative research methods: a guidebook and resource. (3rd.) New York: John Willey.

Testa, F., Iraldo, F. (2010) Shadows and lights of GSCM (Green Supply Chain Management): determinants and effects of these practices based on a multinational study. Journal of Cleaner Production 18 (10 e 11), 953 e 962.

Thomas, W. I. (1927) 1918-1920: The polish peasant in Europe and America. Chicago University Press, New York: Knopf.

Tukker, A., Jansen, B. (2006) Environmental impacts of products: a detailed review of studies. Journal of Industrial Ecology, 10 (3), 159-182.

Tranfield, D., Denyer, D., Smart, P. (2003) Towards a methodology for developing evidenceinformed management knowledge by means of systematic review. British Journal of Management, 14(3), 207-222.

Triviños, A. N. S. (1987) Introdução à pesquisa em ciências sociais. São Paulo: Atlas.

Tylor, E.B. (1871) La ciencia de la cultura. In: Goodenaugh, W. H., Kahn, J.S., Malinowski, A. L., Bronislaw, T. Y. L. O. R., Edward, B. W., \& Leslie, A. (1975) El concepto de cultura: textos fundamentales. Barcelona: Anagrama. 
Ùjhelyi, M. (2001) Az emberi erőforrás menedzsment és fejlesztés, valamint a szervezetfejlesztés kapcsolata (The relationship of HRM, HRM and organisational development). Budapesti Corvinus Egyetem (Corvinus University of Budapest): Ph. D. értekezés.

Vidal-Salazar, M. D., Cordón-Pozo, E., Ferrón-Vilchez, V. (2012) Human resource management and developing proactive environmental strategies: the influence of environmental training and organizational learning. Human Resource Management, November-December, 51(6), 905-934.

Vieira, R.S. (2012) Rio+ 20-Conferência das Nações Unidas sobre meio ambiente e desenvolvimento: contexto, principais temas e expectativas em relação ao novo "direito da sustentabilidade”. Novos Estudos Jurídicos, 17(1), 48-69.

Wals, A.E.J. (2014) Sustainability in higher education in the context of the UN Desd: a review of learning and institutionalization processes. Journal of Cleaner Production, Elsevier Ltd., (62), 8-15.

Wei, Y., \& Miraglia, S. (2017) Organizational culture and knowledge transfer in project-based organizations: theoretical insights from a chinese construction firm. International Journal of Project Management, 35(4), 571-585.

WEF - World Economic Forum. (2016) The Global Risks Report - 2016. Recuperado em: 24 mar.2016, de: https://www.weforum.org/reports/the-global-risks-report-2016. Davos.

Werner, K., Dickson, G., \& Hyde, K. F. (2015) Learning and knowledge transfer processes in a mega-events context: the case of the 2011 Rugby World Cup. Tourism Management, 48, 174-187.

Willis, P., Trondman, M. (2000) Manifesto for ethnography. Ethnography, 1(1), 5-16.

Wilson, L. C. (1977) The use of ethnographic technique in educational research. Review of Educational Research, 47, 245-265.

Wolcott, H. F. (1994) Transforming qualitative data: description, analysis and interpretation. Thousand Oaks, CA: Sage.

World Commission on Environment and Development - Wced. (1987) Our common future. New York: University Press.

Zhigang Li, Yang, F., \& Zhang, D. (2016) The Virtual alliance knowledge sharing model and selection strategy. Procedia Computer Science, 91, 276-283

Zhuge, H. (2006) Knowledge flow network planning and simulation. Decision Support Systems, 42(2), 571-592.

Data da submissão: 09/10/2016

Data de aceite: 03/04/2017 